\title{
UCSF
}

UC San Francisco Previously Published Works

Title

A delayed injection-site reaction in a patient receiving extended-release naltrexone.

Permalink

https://escholarship.org/uc/item/54x1s560

Journal

Substance abuse, 37(2)

ISSN

0889-7077

Authors

Ahamad, Keith

Korthuis, P Todd

Lum, Paula J

et al.

Publication Date

2016-04-01

DOI

10.1080/08897077.2016.1138919

Peer reviewed 


\section{A delayed injection-site reaction in a patient receiving extended-release naltrexone}

\section{Keith Ahamad MD, P. Todd Korthuis MD, MPH, Paula J. Lum MD, Cheyenne Johnson RN \& Evan Wood MD, PhD}

To cite this article: Keith Ahamad MD, P. Todd Korthuis MD, MPH, Paula J. Lum MD, Cheyenne Johnson RN \& Evan Wood MD, PhD (2016) A delayed injection-site reaction in a patient receiving extended-release naltrexone, Substance Abuse, 37:2, 278-280, DOI: $\underline{10.1080 / 08897077.2016 .1138919}$

To link to this article: http://dx.doi.org/10.1080/08897077.2016.1138919

Accepted author version posted online: 28

Jan 2016.

Published online: 28 Jan 2016.

Submit your article to this journal $\pi$

Џ Article views: 55

View related articles $\smile$

View Crossmark data $\nearrow$ 


\title{
A delayed injection-site reaction in a patient receiving extended-release naltrexone
}

\author{
Keith Ahamad, MD ${ }^{\mathrm{a}, \mathrm{b}, \mathrm{c}}$, P. Todd Korthuis, MD, MPH ${ }^{\mathrm{e}, \mathrm{f}}$, Paula J. Lum, MD ${ }^{\mathrm{g}}$, Cheyenne Johnson, $\mathrm{RN}^{\mathrm{a}}$, and \\ Evan Wood, MD, PhD ${ }^{\text {ah }}$

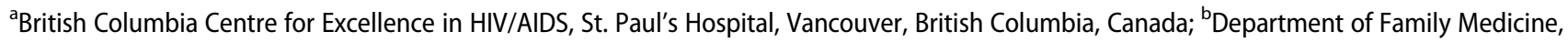 \\ University of British Columbia, Vancouver, British Columbia, Canada; 'Department of Family and Community Medicine, Providence Health Care, \\ Vancouver, British Columbia, Canada; ${ }^{e}$ Department of Medicine, Oregon Health and Science University, Portland, Oregon, USA; ${ }^{f}$ Department of Public \\ Health-Preventive Medicine, Oregon Health and Science University, Portland, Oregon, USA; ${ }^{9}$ Positive Health Program, HIV/AIDS Division, University of \\ California, San Francisco, California, USA; ${ }^{\mathrm{h}}$ Department of Medicine, University of British Columbia, Vancouver, British Columbia, Canada
}

\section{ABSTRACT}

Background: Pharmacotherapy, such as oral naltrexone, has proven effective in treating alcohol use disorder, although medication adherence has presented challenges. Although a formulation of extendedrelease naltrexone for intramuscular injection has been developed to counter daily adherence issues, injection-site reactions can occur within days of depot injection. Case: The authors report a case of an individual with alcohol use disorder who had a previously undescribed delayed injection-site reaction that occurred 11 days after injection. Subsequent challenge with the medication resulted in recurrence of the reaction. Discussion: Although extended-release naltrexone is generally well tolerated, injection-site reactions can complicate treatment and can appear more than 10 days after medication administration.

\section{KEYWORDS}

Alcohol use disorder; extended-release naltrexone; injection-site reaction

\section{Introduction}

Alcohol use disorder (AUD) is associated with health and social harms. In the United States alone, the economic impact from excessive alcohol consumption in 2006 was an estimated $\$ 223.5$ billion. $^{1}$ A number of evidence-based pharmacologic therapies exist for the treatment of AUD. A recent meta-analysis of clinical trials suggests that acamprosate and oral naltrexone are 2 of the most effective agents for treating AUD. ${ }^{2}$ Oral naltrexone decreases rates of return to heavy drinking, with the number needed to treat (NNT) on the order of 9 to $12^{2,3}$; however, adherence to daily dosing is a major challenge to successful AUD treatment. ${ }^{4}$

Extended-release naltrexone (XR-NTX), injected intramuscularly once monthly, was approved by the US Food and Drug Administration (FDA) for treatment of AUD in 2006. ${ }^{5}$ It improves AUD treatment adherence in difficult-to-treat populations, leading to reductions in alcohol consumption and consequences related to alcohol use, and decreased health care costs for those who persist with treatment. ${ }^{6,7}$ A meta-analysis from 2014 showed that XR-NTX has its strongest influence on decreasing number of heavy drinking days when compared with placebo, although only 3 studies were included in the analysis, evidence that research efforts need to continue. ${ }^{2}$

Although XR-NTX has generally proven safe for the treatment of AUD, it can present certain challenges with tolerability or unforeseen side effects. One challenge with injectable medications is injection-site complication. In 2008, the FDA issued an alert for the XR-NTX formulation, known as Vivitrol, specific to this issue. At that time, there were 196 reports of injection-site reactions that included "cellulitis, induration, hematoma, abscess, sterile abscess, and necrosis," 16 of which needed surgical intervention. ${ }^{8}$ Consistent with the Vivitrol product monograph, the alert warned against injecting into subcutaneous fat and recommended using the 1.5- or 2.0-inch, 20 -gauge needles that come with the kit. Typically, these reactions occur within 1 to 3 days of injection.

The present case describes, to our knowledge, a previously unreported type of delayed injection-site reaction to XR-NTX in a human immunodeficiency virus (HIV)-positive individual with severe AUD provided with XR-NTX while enrolled in a clinical trial. Written consent was obtained from the patient.

\section{Case presentation}

A 41-year-old, HIV-positive male with a history of depression, anxiety, and severe alcohol use disorder was enrolled in the National Drug Abuse Treatment Clinical Trials Network (CTN) study Comparing Treatments for HIV-Infected Opioid and Alcohol Users in an Integrated Care Effectiveness Study (CHOICES CTN-0055). Medications included co-formulated tenofovir-emtricitabine, atazanavir, and ritonavir. The patient reported daily drinking from the age of 10, 6 to 30 beers per day, a family history of alcohol addiction, strong cravings for alcohol, and multiple severe health and social consequences related to his addiction. There was no history of complicated withdrawal (seizures or delirium tremens), and no previously prescribed medication or psychosocial treatment to treat his severe AUD. There was no history of opioid use disorder. At study enrolment, 
the patient reported no alcohol for the previous 5 days and was started on off-label gabapentin to manage ongoing anxiety and cravings, presumed secondary to protracted withdrawal. ${ }^{9}$ Baseline laboratory findings included CD4 absolute 660 cells $/ \mu \mathrm{L}$, hepatitis $\mathrm{B}$ surface antigen negative, hepatitis $\mathrm{C}$ antibody negative, HIV viral load $<40$ copies $/ \mathrm{mL}$, white blood count $7.6 \times$ $10^{9} / \mathrm{L}$, platelet count $208 \times 10^{9} / \mathrm{L}$, international normalized ratio (INR) 0.9, alanine aminotransferase (ALT) $58 \mathrm{U} / \mathrm{L}$, and aspartate aminotransferase (AST) $46 \mathrm{U} / \mathrm{L}$. He was subsequently randomized to XR-NTX. Of note, his body habitus, in particular the gluteal muscle, did not exhibit any contraindications or challenges for XR-NTX injection (e.g., obesity).

Following his first injection with XR-NTX into his left buttock, the patient had no immediate reaction or concerns for almost 2 weeks. However, on day 16 post injection, the individual contacted the study team regarding new and ongoing swelling of his left buttock in the vicinity of the XR-NTX injection. At this time, the patient stated that the swelling started while sitting in a car for 12 hours, 11 days following his injection of XR-NTX. Subsequently, the patient's injection site area was very swollen and putting on jeans was difficult for 2 days. At that time, he reported that the affected area was warm to touch, but denied history of pain or constitutional symptoms, including fever. The patient used icepacks during that time to treat the swelling. On examination, 17 days after the first injection, the patient looked well and vital signs were normal. The affected area was warm to touch and slightly swollen when compared with his right buttock, with no difference in color and no tenderness on palpation. There was no appreciable mass on palpation. The rest of the physical examination was unremarkable. As per the patient's preference and based on a reassuring physical examination, no further investigations were done at that time.

Thirty-four days following the first XR-NTX injection, the patient received a second injection in the right buttock. The decision to give a second injection was based on the atypical initial injection reaction and the patient's desire to continue XR-NTX due to decreased alcohol craving following the initial injection. Laboratory results drawn on the day of the second injection revealed white blood cells $7.6 \times 10^{9} / \mathrm{L}$; hemoglobin (Hb) $149 \mathrm{~g} / \mathrm{L}$; platelet count $215 \times 10^{9} / \mathrm{L}$; and absolute CD4 630 cells $/ \mu \mathrm{L}$. The patient was asked to return to the clinic if any signs of swelling occurred. On day 2 following the injection, he returned with complaints of swelling on this right buttock that started 24 hours after the injection. At this time, he reported pain in the right buttock, temperature of $39^{\circ} \mathrm{C}$, and nausea, which was well controlled with dimenhydrinate $50 \mathrm{mg}$ every 4 hours, as needed. On examination, he looked well, was in no distress, and was afebrile and the rest of his vitals were normal. His right buttock was swollen and extending to his lower back. The buttock was warm and tender to palpation, slightly erythematous. No mass, nor area of fluctuance, was palpable. The rest of his physical examination was unremarkable. The report of an ultrasound, performed 2 days following his second injection, read "edema in the soft tissue of the right buttock extending from the mid lower back to the right lateral hip. No discrete abscess is identified."

Three days following the second injection, he was seen at a community clinic and encouraged to return to the hospital for a repeat ultrasound and blood work. On day 4 , he was seen by a community outreach nurse who reported that the swelling had decreased and the pain was well controlled with ibuprofen $400 \mathrm{mg}$ every 6 hours and applied ice packs. The outreach nurse followed him daily and reported daily improvements in pain and swelling. The patient followed up for a study visit 4 weeks post injection. He reported that the swelling started to decrease 8 days after the second injection. The patient denied any signs or symptoms consistent with a systemic allergic reaction.

As the patient reported a significant reduction in craving for alcohol, total drinking days, and heavy drinking days while on XR-NTX, he desired to continue with medication. The study treatment team viewed the reaction to be most consistent with the injection suspension itself, rather than naltrexone per se. After reviewing risks and benefits of repeat naltrexone exposure with the patient, he was transitioned to oral naltrexone with no further complication and continues to experience decreased cravings and psychosocial improvement as per follow-up with his clinical providers 5 months following switch to oral naltrexone.

\section{Discussion}

We have described a case of an individual who had a delayed injection-site reaction to XR-NTX who, when given a subsequent injection at a different site, had a worse reaction with faster onset. Given the individual's significant decrease in alcohol use and craving for alcohol, and his desire to continue on medication, he was successfully transitioned to oral naltrexone without complication.

Clinical trials and postmarketing surveillance suggest that XR-NTX is safe, well tolerated, and effective in treating AUD. ${ }^{2}$ Injection-site reactions are typically minor and, in most cases, attributed to inappropriate injection of the medication (i.e., into subcutaneous fat rather than intramuscularly). ${ }^{10}$ Due to the potential for infection when depot medications are used, the FDA released a postmarket drug and safety "Information for Healthcare Professionals" notice in 2008 suggesting clinicians monitor for "pain, swelling, tenderness, induration, bruising, pruritus, or redness at the injection site that does not improve or worsens within two weeks."

The present case presents an interesting scenario where a patient had a delayed injection-site reaction that did not appear for 11 days post injection, and, when reexposed to XR-NTX at a different location, had a similar more severe reaction with rapid onset with signs of systemic involvement, suggestive of a possible hypersensitivity reaction. The patient tolerated a switch to oral naltrexone without difficulty, suggesting that the reaction was not due to naltrexone itself. XR-NTX uses a microsphere technology to deliver its sustained-release formulation. ${ }^{11}$ We hypothesize that this individual experienced what is likely a rare and previously unreported delayed hypersensitivity reaction to the microspheres. Although the diluent (diluent ingredients: carboxymethylcellulose sodium salt, polysorbate 20 , sodium chloride, and water for injection) could have caused a similar reaction, this would have been expected to dissipate by 11 days following the initial injection, but the microspheres persist for several weeks. It should be noted, however, that HIV itself cannot be ruled out as a contributor, as adverse drug reactions have been reported at higher rates in HIV-positive 
patients. Abacavir hypersensitivity reaction has been well described, with symptoms occurring between 9 and 11 days following drug exposure and reoccur, similar to our patient, when rechallenged. ${ }^{12}$ Patients should be monitored closely following XR-NTX injection, and monitoring the time to injecting-site reaction should be included in postmarketing surveillance. Rarely, localized reactions may indeed occur without infectious etiology, which may be related to HIV. This may not preclude prescribing the oral form of naltrexone as in this individual.

\section{Funding}

The study was supported by the US National Institutes of Health (UG1 DA015815 and R25 DA037756) and the Canadian Institutes of Health Research through the Canadian Research Initiative on Substance Misuse (SMN-139148). This research was undertaken, in part, thanks to funding for a Tier 1 Canada Research Chair in Inner City Medicine, which supports Dr. Evan Wood. The authors declare that they have no competing interests. Dr. Korthuis serves as principal investigator of 2 studies funded by the National Institute on Drug Abuse that receive XR-NTX study medication donated by Alkermes.

\section{Author contributions}

Keith Ahamad and Evan Wood prepared the first draft of the manuscript. All coauthors contributed to the drafting of the final manuscript.

\section{References}

[1] Bouchery EE, Harwood HJ, Sacks JJ, Simon CJ, Brewer RD. Economic costs of excessive alcohol consumption in the U.S., 2006. Am J Prev Med. 2011;41:516-524.
[2] Jonas DE, Amick HR, Feltner C, et al. Pharmacotherapy for adults with alcohol use disorders in outpatient settings: a systematic review and meta-analysis. JAMA. 2014;311:1889-1900.

[3] Maisel NC, Blodgett JC, Wilbourne PL, Humphreys K, Finney JW. Meta-analysis of naltrexone and acamprosate for treating alcohol use disorders: When are these medications most helpful? Addiction. 2013;108:275-293.

[4] Swift R, Oslin DW, Alexander M, Forman R. Adherence Monitoring in Naltrexone Pharmacotherapy Trials: A Systematic Review*. Journal of studies on alcohol and drugs. 2011 Nov;72 (6):1012-8.

[5] Warren KR, Hewitt BG. NIAAA: Advancing alcohol research for 40 years. Alcohol Health \& Research World. 2010 Jan 1;33(1):5.

[6] Collins SE, Grazioli VS, Torres NI, et al. Qualitatively and quantitatively evaluating harm-reduction goal setting among chronically homeless individuals with alcohol dependence. Addict Behav. 2015;45:184-190.

[7] Bryson WC, McConnell KJ, Korthuis PT, McCarty D. Extendedrelease naltrexone for alcohol dependence: persistence and healthcare costs and utilization. The American journal of managed care. 2011 Jun;17(Suppl 8):S222.

[8] Food and Drug Administration. Information for Healthcare Professionals: Naltrexone Injection Site Reactions (Naltrexone for ExtendedRelease Injectable Suspension, marketed as Vivitrol). Rockville, MD: Food and Drug Administration; 2008.

[9] Mason BJ, Quello S, Goodell V, Shadan F, Kyle M, Begovic A. Gabapentin treatment for alcohol dependence: A randomized clinical trial. JAMA Intern Med. 2014;174:70-77.

[10] Gastfriend DR. Intramuscular extended-release naltrexone: Current evidence. Ann N Y Acad Sci. 2011;1216:144-166.

[11] Goonoo N, Bhaw-Luximon A, Ujoodha R, Jhugroo A, Hulse GK, Jhurry D. Naltrexone: A review of existing sustained drug delivery systems and emerging nano-based systems. J Controlled Release. 2014;183:154-166.

[12] Stokes SC, Tankersley MS. HIV: Practical implications for the practicing allergist-immunologist. Ann Allergy Asthma Immunol. 2011;107:1-9. 